\title{
Research participants in NGS studies want to know about incidental findings
}

\author{
Anne Marie Jelsig ${ }^{\star, 1}$, Niels Qvist ${ }^{2}$, Klaus Brusgaard ${ }^{1}$ and Lilian Bomme Ousager ${ }^{1}$ \\ Following the implementation of high-throughput sequencing legal and ethical issues are discussed intensively. The management \\ of incidental findings (IFs) in a research setting have been investigated but there is a lack of literature concerning research \\ participant's perspective. The aim of this study was to investigate whether research participants want disclosure of IFs and what \\ kind of IFs they want to know about. One hundred and twenty-seven research participants in a study of gastrointestinal polyps \\ were informed about whole-exome sequencing and the risk of IFs. They were asked to decide whether they (A) wanted disclosure \\ of IFs no matter whether the variants were associated with a non-treatable or non-preventable condition, (B) wanted disclosure of \\ variants associated with treatable or preventable conditions or (C) wanted no disclosure at all. Participants who wanted \\ disclosure of all the IFs (A) accounted for the majority $(n=78), 45$ of the participants only wanted disclosure of variants, which \\ could lead to surveillance or treatment (B) and 4 participants did not want IFs to be disclosed at all (C). The study showed that \\ almost all research participants wanted disclosure of at least some types of IFs.
}

European Journal of Human Genetics (2015) 23, 1423-1426; doi:10.1038/ejhg.2014.298; published online 21 January 2015

\section{INTRODUCTION}

The rapid development in genetic techniques such as massive parallel sequencing (next-generation sequencing, NGS) has challenged both clinical and research practice concerning the management of incidental findings (IFs). Especially the possibility of whole-exome sequencing (WES) and whole-genome sequencing (WGS) has raised legal and ethical questions such as how and when to disclose IFs to the patient/participant. ${ }^{1-6}$

IFs - also named as secondary findings - may be defined as: 'a finding concerning an individual research participant that has potential health or reproductive importance and is discovered in the course of conducting research but is beyond the aims of the study. ${ }^{77}$ In genetics, IFs are typically thought of as variants that are of significance to the patient/participant or his/her family but is not as such relevant to the research project or the clinical situation. However, the definition of IFs is still not clear and the discussion of when a finding is 'incidental' is ongoing.

The frequency of IFs is unknown and will to some extent depend on the study design. In a clinical study of 250 patients, (mainly children with an undiagnosed neurological phenotype) actionable (clinically relevant) IFs were found in 30 patients (12\%) and the carrier status of autosomal recessive disorders were found in 13 patients $(5,2 \%){ }^{8}$

The American Society of Human Genetics (ASHG) published their guidelines concerning clinical management of IFs, when performing exome/large-scale sequencing in a diagnostic set-up. ${ }^{9}$ ASHG recommends that a list of genes should be investigated and that constitutional variants found in these genes should be reported back. ${ }^{9}$ The recommendations have been followed by an intense debate $3,4,10-12$

IFs have been discussed in a research setting; ${ }^{7,13-16}$ both IFs in general, ${ }^{7}$ whether one should disclose IFs or not ${ }^{14}$ and IFs from a researcher's perspective. ${ }^{15,16}$ Klitzman et al ${ }^{16}$ interviewed researchers, and the majority believed that research participants should have the option to receive at least some incidental genetic research results.

The present study focuses on the research participants' attitude towards IFs. The aim was to investigate whether research participants wanted IFs disclosed and what kind of IFs they wanted disclosed.

\section{MATERIALS AND METHODS}

\section{Participants}

Research participants were recruited from the Region of Southern Denmark, which accounts for $1 / 5$ of the Danish population. The population is representative of the Danish population concerning socioeconomic status and ethnical background. Danish citizens are government insured in case of illness or in the case of need of medical evaluations or treatment. The overall study aimed to analyse the DNA from blood samples of patients with benign gastrointestinal (GI) polyps with NGS. The Committee of Health Research Ethics approved the study.

The initial inclusion criteria were a histopathological diagnosis of one or more hamartomatous polyps in the GI-tract. The participants were identified in the Danish National Pathological Register where all histopathological diagnoses made in Denmark are registered. Participants between 18 and 80 years were included. Three hundred and thirty-eight potential participants where identified and contacted in writing and were invited to have further information on the research project and genetic analysis. One hundred and forty-two participants responded.

The majority of the responders considered themselves healthy and did not suspect genetic conditions in their families. They did not follow any gastrointestinal surveillance programme, because a solitary hamartomatous polyp is generally not considered to be a risk factor for later malignancy. Four participants were, however, known carriers of disease-causing variants: one with a $\mathrm{MSH} 2$ variant, one with a

\footnotetext{
${ }^{1}$ Department of Clinical Genetics, Odense University Hospital, Odense C, Denmark; ${ }^{2}$ Surgical Department A, Odense University Hospital, Odense C, Denmark

*Correspondence: Dr AM Jelsig, Department of Clinical Genetics, Odense University Hospital, Sdr Boulevard 29, 5000 Odense C, Denmark. Tel: +45 65411725; Fax: +45 6541 4875; E-mail: anne.marie.jelsig@rsyd.dk

Received 6 April 2014; revised 17 December 2014; accepted 19 December 2014; published online 21 January 2015
} 
SMAD4 variant, one with an APC variant and one woman who was a carrier of X-linked Duchenne muscular dystrophy. In addition, one patient was known to have adult polycystic kidney disease but had not been genetically tested. The group consisted mainly of Caucasians of Danish origin, with one emigrant from France, one from Canada and one from Turkey. Every participant was informed about WES and the possibility of detecting IFs. The participants could decide whether they wanted the information by telephone or outpatient consultations, which both lasted around 40 min per participant. Fourteen chose to come in for consultations. All, but one, were informed by the same physician trained in clinical genetic counselling.

Each participant was informed about the risk of detecting IFs and that this presumably was low, but that the likelihood is unknown. Concerning disclosure of IFs, the participants were presented with three options:

- To receive disclosure of all the IFs, that might be found in the project - even if the variant might lead to risk of an untreatable or unpreventable disease (group A).

- To receive disclosure on IFs only if the variant might lead to a condition that is treatable, preventable or for which there can be an offered surveillance (group B).

- Not to receive disclosure of IFs at all (group C).

The participants were given time to consider the choices for $\sim 1$ month before making their final decision and they had the possibility of writing (email) or calling the researcher for additional information in this period. The researcher did not contact the participants in this period. The consultations were 'semi-structured' giving the same information on genetic testing and the same examples of actionable variants (heart conditions and cancer genes) and nontreatable conditions (neurodegenerative disorders such as dementia) to all participants. Furthermore, the issues concerning information to family members and genetic testing of children were discussed in a similar way with every participant. The risk of finding variants that are difficult to interpret with our present knowledge (variants of unknown significance) was also discussed. It was also stated that there would be no follow-up on the generated data outside of the research period of 3 years, however, if the participants choose to be informed about IFs (whether they were actionable or not) they would be offered genetic counselling in the case of detection of a disease-causing variants. Arguments for and against disclosure were discussed, and especially the psychological aspect of learning oneself 'to be at risk' even though nothing can be done was discussed. Every participant was encouraged to decide what was right for him/her.

\section{Statistics}

Fisher's exact test was used for comparison of age and sex of the participants and their choices on the three options for IFs.

\section{RESULTS}

A total of 127 participants (74 females and 53 males) consented to report their decision. The remaining 15 participants were excluded from the project for various reasons: 12 did not respond, 1 declined participation due to concerns on life insurance issues and 1 was diagnosed with cancer and did not want to participate.

The majority of participants wanted disclosure of all IFs (A; $n=78$ $(61 \%))$. Forty-five participants wanted disclosure of actionable variants $(\mathrm{B} ; 36 \%)$ and four participants $(3 \%)$ did not want to receive information on IFs at all (C; Figure 1). The average age of participation was 58 years (females $=55$ years, males $=61$ years; Table 1). There was no significant difference in the answers relating to sex $(P=0.3)$ or the various age groups of the participants $(18-29$ years, 30-39 years, 40-49 years, 50-59 years, 60-69 years and 70-80 years; $P=0.4$ ).

The four patients, who were known with a disease-causing variant and who had received genetic counselling earlier, all wanted disclosure of all IFs.

\section{DISCUSSION}

This study shows that most research participants are interested in disclosure of IFs. The majority (61\%) wanted disclosure of IFs even if the variants cause non-treatable and non-preventable conditions. However, it is notable that a large part of the participants $(36 \%)$ only want actionable IFs to be disclosed. Only a few participants in this study did not want IFs to be disclosed at all (3\%). There were no significant differences between males and females in their answers. For most of the participants in this study it was not a question whether they wanted disclosure or not, but what type of variants they wanted to be disclosed.

Although many researchers and clinicians may find that the benefits of disclosure IFs outweigh the drawbacks, several arguments against disclosure have been raised. ${ }^{17}$ In a review of IFs Lohn et al ${ }^{17}$ have listed arguments against the disclosure of IFs as discussed by others.

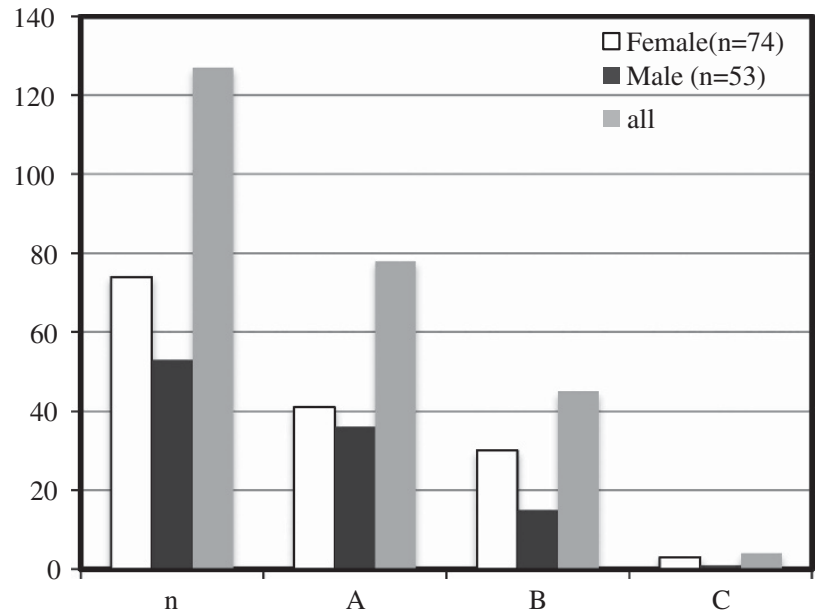

Figure 1 Research-participants answers concerning disclosure of IFs. A, disclosure of all IFs, B, disclosure of actionable variants, C, no disclosure of IFs, $n$, number of participants.

Table 1 The answers of females and males in the different age groups

\begin{tabular}{lrrrrrr}
\hline Age (years) & $n$ & Female & Male & $A$ & $B$ & $C$ \\
\hline $18-29$ & 8 & 6 & 2 & 3 & 4 & 1 \\
$30-39$ & 9 & 6 & 3 & 6 & 3 & 0 \\
$40-49$ & 13 & 10 & 3 & 8 & 4 & 1 \\
$50-59$ & 26 & 17 & 9 & 15 & 9 & 2 \\
$60-69$ & 48 & 28 & 20 & 30 & 18 & 0 \\
$70-80$ & 23 & 7 & 16 & 16 & 7 & 0 \\
All & 127 & 74 & 53 & 78 & 45 & 4
\end{tabular}

Abbreviations: A, disclosure of all IFs; $\mathrm{B}$, disclosure of actionable variants; $\mathrm{C}$, no disclosure of IFs; $n$, number of participants. 
Arguments such as the psychological harm to patients, the disclosure of non-paternity, disclosure of variants of unknown significance, as well as resources required to interpret, communicate and follow-up on IFs are mentioned. However, the authors conclude that non-disclosure may be unethical and that an intermediate disclosure policy between full and non-disclosure may be considered to guide disclosure decisions. ${ }^{17}$ Most of these arguments are based on theoretical considerations and were not derived from studies of research participants. A systematic review on the ethical reflections on IFs was also conducted by Christenhusz et al ${ }^{18}$ Considerations of WES in minors and the suggestion that parents could decide to learn about the risk for adult-onset diseases in their children have been raised as risks to an open future. The psychological impact for children and parents is unknown but could be considerable.

Some studies similar to this have been published: Facio et al ${ }^{19}$ conducted a survey on 311 individuals. They were asked about their preference for knowing IFs in certain categories: actionable variants, non-actionable variants, gene variants with importance for the participants' family but not himself and uncertain gene variants. The study found that participants' attitudes towards receipt of information were highly positive for all the four categories. The authors also found that their results reflected the ability of the participants to discriminate between different types of genetic results. ${ }^{19}$ Shahmirzadi et al ${ }^{20}$ conducted a retrospective study on the patients' decisions to learn IFs when undergoing clinical WES. In this study, the options for IFs where divided into carrier status of recessive disorders, predisposition to late-onset diseases, predisposition to increased cancer risk and early-onset diseases. The majority (93.5\%) of their patients chose to receive information on IFs for one or more available categories. Sapp et $a^{21}$ conducted semi-structured interviews with 25 parents of 13 minor probands enrolled in a study on the cause of rare genetic conditions. Parents were asked to discuss their preferences to receive different types of results from the exome sequencing. Many of the parents preferred to receive all types of results, but had reservations about learning about the predispositions for untreatable adult-onset conditions and carrier status for recessive conditions.

\section{LIMITATIONS}

Numerous factors such as previous experience with genetics, serious illness, having children, personality traits and psychological states and so on, could influence decision-making. In our study, the large majority of participants were healthy and did not suspect a genetic condition in their family. Research participants could be more motivated to learn about the results as they have already agreed to participate in the research, as such there may be a bias reflected in our results from the persons who are more likely to want to know. Our $42 \%$ response rate leaves open the possibility that non-responders have different - perhaps more reluctant attitudes towards IFs.

In a recent paper on biobanks and IFs, Viberg et al ${ }^{22}$ argue about the importance of a distinction between incidental discovered disease and an incidental discovered increased genetic risk for diseases of unclear predictive value. They call for empirical studies on how the latter will be explained to participants. Our study sought to convey the complexity of WES results and the potential for identifying IFs that are difficult to interpret before assessing the results of preferences for learning.

There are some limitations to this study and its design: Most importantly the study did not collect data on the motivations for the participants choices or to what extent the research participants understood the somewhat complex information they where given. Furthermore, we have not planned any follow-up on the participants view after returning the IFs. In a new study, a more systematic gathering of participants' thoughts before, during and after WES would, therefore, be relevant.

This study does not deal with the issue on whether the researchers have an ethical or legal obligation to look for IFs. This discussion is relevant, however, especially as the ASHG's clinical guidelines recommend that a list of genes should be investigated (eg APC, BRCA1 and so on) and that the constitutional variants found in these genes 'should be reported by the laboratory to the ordering clinician, regardless of the indication for which the clinical sequencing was ordered.'

We do feel that there is a distinction between a research setting and a clinical setting, although the distinction may not always be crystal clear. The purpose of research is often not to reveal a diagnosis of a single patient, but to investigate a whole group in order to answer a hypothesis and create new ones. WES/WGS is used in basic research to reveal the underlying molecular differences, for example, in tumours, and the purpose is not necessarily to detect a pathogenic germline variant with coverage sufficient for diagnostics use. Thus, the management of IFs could be different in these settings as the reason for the use of WES/WGS is different.

\section{CONCLUSION}

Among our study responders, the majority wanted to learn about IFs. Our findings can be used to contribute to the discussion of IFs in a research setting, suggesting a positive attitude towards learning results. Participants demonstrated their ability to discriminate between the different types of genetic information. We suggest that the options for reporting IFs in research studies be incorporated in the consent form. In addition, we think that IFs can be discussed in a shared decisionmaking encounter and that participants can make informed choices whether they prefer to receive disclosure of IFs or not.

\section{CONFLICT OF INTEREST}

The authors declare no conflict of interest.

1 Ormond KE, Wheeler MT, Hudgins L et al: Challenges in the clinical application of whole-genome sequencing. Lancet 2010; 375: 1749-1751.

2 Gliwa C, Berkman BE: Do researchers have an obligation to actively look for genetic incidental findings? Am J Bioeth 2013; 13: 32-42.

3 Evans BJ: Minimizing liability risks under the ACMG recommendations for reporting incidental findings in clinical exome and genome sequencing. Genet Med 2013; 15: 915-920.

4 McGuire AL, Joffe S, Koenig BA et al: Point-counterpoint. Ethics and genomic incidental findings. Science 2013; 340: 1047-1048.

5 Biesecker LG: Opportunities and challenges for the integration of massively parallel genomic sequencing into clinical practice: lessons from the ClinSeq project. Genet Med 2012; 14: 393-398.

6 Biesecker LG: Incidental variants are critical for genomics. Am J Hum Genet 2013; 92 : 648-651.

7 Wolf SM, Lawrenz FP, Nelson CA et al: Managing incidental findings in human subjects research: analysis and recommendations. J Law Med Ethics 2008; 36: 219-248, 211.

8 Yang Y, Muzny DM, Reid JG et al: Clinical whole-exome sequencing for the diagnosis of mendelian disorders. N Engl J Med 2013; 369: 1502-1511.

9 Green RC, Berg JS, Grody WW et al: ACMG recommendations for reporting of incidental findings in clinical exome and genome sequencing. Genet Med 2013; 15: 565-574.

10 Burke W, Matheny Antommaria AH, Bennett R et al: Recommendations for returning genomic incidental findings? We need to talk!. Genet Med 2013; 15: 854-859.

11 Wolf SM, Annas GJ, Elias S: Point-counterpoint. Patient autonomy and incidental findings in clinical genomics. Science 2013; 340: 1049-1050.

12 Allyse M, Michie M: Not-so-incidental findings: the ACMG recommendations on the reporting of incidental findings in clinical whole genome and whole exome sequencing. Trends Biotechnol 2013; 31: 439-441.

13 Jackson L, Goldsmith L, O'Connor A, Skirton $\mathrm{H}$ : Incidental findings in genetic research and clinical diagnostic tests: a systematic review. Am J Med Genet A 2012; 158A: 3159-3167.

14 Steinsbekk KS, Solberg B: Should genetic findings from genome research be reported back to the participants? Tidsskr Nor Laegeforen 2012; 132: 2190-2193. 
15 Meacham MC, Starks H, Burke W, Edwards K: Researcher perspectives on disclosure of incidental findings in genetic research. J Empir Res Hum Res Ethics 2010; 5: 31-41.

16 Klitzman R, Appelbaum PS, Fyer A et al: Researchers' views on return of incidental genomic research results: qualitative and quantitative findings. Genet Med 2013; 15: 888-895.

17 Lohn Z, Adam S, Birch PH, Friedman JM: Incidental Findings from Clinical GenomeWide Sequencing: A Review. J Genet Couns 2013; 23: 463-473.

18 Christenhusz GM, Devriendt K, Dierickx K: To tell or not to tell? A systematic review of ethical reflections on incidental findings arising in genetics contexts. Eur J Hum Genet 2013; 21: 248-255.
19 Facio FM, Eidem H, Fisher T et al: Intentions to receive individual results from whole genome sequencing among participants in the ClinSeq study. Eur J Hum Genet 2013; 21: 261-265.

20 Shahmirzadi L, Chao EC, Palmaer E, Parra MC, Tang S, Gonzalez KD: Patient decisions for disclosure of secondary findings among the first 200 individuals undergoing clinical diagnostic exome sequencing. Genet Med 2014; 16: 395-399.

21 Sapp J, Dong D, Stark C et al: Parental attitudes, values, and beliefs toward the return of results from exome sequencing in children. Clin Genet 2014; 85: 120-126.

22 Viberg J, Hansson MG, Langenskiold S, Segerdahl P: Incidental findings: the time is not yet ripe for a policy for biobanks. Eur J Hum Genet 2014; 22: 437-441. 